\title{
Putative alternative translation start site- encoding nucleotides of CPR5 regulate growth and resistance
}

\author{
Muhammad B. Faisal ' (D), Tsanko S. Gechev² (D), Bernd Mueller-Roeber ${ }^{2,3}$ (D) and Paul P. Dijkwel ${ }^{1 *}$ (D)
}

\begin{abstract}
Background: The Arabidopsis CONSTITUTIVE EXPRESSER of PATHOGENESIS-RELATED GENES 5 (CPR5) has recently been shown to play a role in gating as part of the nuclear pore complex (NPC). Mutations in CPR5 cause multiple defects, including aberrant trichomes, reduced ploidy levels, reduced growth and enhanced resistance to bacterial and fungal pathogens. The pleiotropic nature of cpr5 mutations implicates that the CPR5 protein affects multiple pathways. However, little is known about the structural features that allow CPR5 to affect the different pathways.

Results: Our in silico studies suggest that in addition to three clusters of putative nuclear localization signals and four or five transmembrane domains, CPR5 contains two putative alternative translation start sites. To test the role of the methionine-encoding nucleotides implicated in those sites, metCPR5 CDNAs, in which the relevant nucleotides were changed to encode glutamine, were fused to the CPR5 native promoter and the constructs transformed to cpr5-2 plants to complement cpr5-compromised phenotypes. The control and metCPR5 constructs were able to complement all cpr5 phenotypes, although the extent of complementation depended on the specific complementing plant lines. Remarkably, plants transformed with metCPR5 constructs showed larger leaves and displayed reduced resistance when challenged to Pseudomonas syringae pv Pst DC3000, as compared to control plants. Thus, the methionine-encoding nucleotides regulate growth and resistance. We propose that structural features of the CPR5 N-terminus are implicated in selective gating of proteins involved in regulating the balance between growth and resistance.

Conclusion: Plants need to carefully balance the amount of resources used for growth and resistance. The Arabidopsis CPR5 protein regulates plant growth and immunity. Here we show that N-terminal features of CPR5 are involved in the regulation of the balance between growth and resistance. These findings may benefit efforts to improve plant yield, while maintaining optimal levels of disease resistance.
\end{abstract}

Keywords: CPR5, Plant growth, Disease resistance, Cell death, Arabidopsis thaliana, Endoreduplication

\section{Background}

Plants recognise attack of most pathogens and respond through the production of antimicrobial proteins and the activation of defence responses [1]. In an attempt to define the signalling processes leading to plant disease resistance and susceptibility, a variety of mutants were isolated that

\footnotetext{
* Correspondence: p.dijkwel@massey.ac.nz

${ }^{1}$ School of Fundamental Sciences, Massey University, Palmerston North, New Zealand

Full list of author information is available at the end of the article
}

displayed a defective resistance response. For example, mutants with hyper-susceptibility phenotypes e.g., npr1 for nonexpresser of pathogenesis related genes1 [2-4] and eds5 for enhanced disease susceptibility $5[5,6]$ and mutants that show constitutive activation of defence-related genes and enhanced defence responses (e.g., acd6 (accelerated cell death6), cpr1 (constitutive expresser of pathogenesis-related genes1) [7], cpr2 and cpr6 [8]) were isolated. In 1997, Bowling and coworkers reported the cpr5-2 mutant, which displayed constitutive expression of pathogenesis-related genes PR1, PR5 and

(c) The Author(s). 2020 Open Access This article is licensed under a Creative Commons Attribution 4.0 International License, which permits use, sharing, adaptation, distribution and reproduction in any medium or format, as long as you give appropriate credit to the original author(s) and the source, provide a link to the Creative Commons licence, and indicate if changes were made. The images or other third party material in this article are included in the article's Creative Commons licence, unless indicated otherwise in a credit line to the material. If material is not included in the article's Creative Commons licence and your intended use is not permitted by statutory regulation or exceeds the permitted use, you will need to obtain permission directly from the copyright holder. To view a copy of this licence, visit http://creativecommons.org/licenses/by/4.0/ The Creative Commons Public Domain Dedication waiver (http://creativecommons.org/publicdomain/zero/1.0/) applies to the data made available in this article, unless otherwise stated in a credit line to the data. 
PDF1.2, aberrant trichomes and hypersensitive response (HR)-like lesions on their leaves in the absence of any pathogen attack [9]. Later, Jing et al. [10] described the old1 (onset of leaf death1) mutant that showed HR-like lesions, early leaf senescence and the early onset of leaf death. In the same year, Yoshida et al. [11] reported hys1 (hypersenescence1) as a mutant with accelerated leaf senescence and hypersensitivity to sugar. Borghi et al. [12] isolated cpr5-3, which showed reduced leaf $\mathrm{K}^{+}$levels and cpr5-2 phenotypes. CPR5, OLD1 and HYS1 were all found to be the same locus and subsequent studies showed that cpr5 plants also exhibit hypersensitivity to exogenous abscisic acid (ABA), jasmonic acid (JA) and ethylene (ET) [13-15]. The pleiotropic nature of cpr5 mutants suggests that CPR5 is a master regulator of different cellular pathways [16].

The functional analyses of the CPR 5 protein have been difficult because this protein is only found in plants and the moss, Physcomitrella patens [13]. Nevertheless, computational studies showed that CPR5 is predicted to contain an N-terminal bipartite nuclear localisation signal (NLS) and multiple transmembrane (TM) domains at the carboxyl terminus [11], which is consistent with the model that CPR5 is a nuclear membrane protein [14]. Recently, CPR5 has emerged as one of the nucleoporins and shown to function as part of the nuclear pore complex (NPC) $[14,15,17]$. CPR5 was suggested to participate in NPC gating through the formation of CPR5CPR5 homodimers and deregulated nucleocytoplasmic transport as a consequence of mutations in the CPR5 gene was proposed to result in compromised immunity in cpr5 mutants. However, it is not clear how structural features of the CPR 5 proteins mediate selectivity.

In silico studies suggest that CPR5 contains three clusters of NLS-encoding residues, two clusters of Casein Kinase (CK) phosphorylation sites and a number of alternative translation start sites. Here, we mutated putative alternative translation start site-encoding residues in order to test their role in CPR5 function. The mutated genes were used to complement the cpr5-2 mutant. We found that the complementing lines changed growth and disease resistance against Pst DC3000 demonstrating that the CPR5 $\mathrm{N}$-terminus is involved in the regulation of plant growth and disease resistance.

\section{Results}

\section{CPR5 is predicted to contain multiple translation initiation sites}

The CPR5 transcript possess multiple in-frame start codons at positions $\mathrm{a}, \mathrm{b}$, and $\mathrm{c}$ as shown in Fig. 1. If these are functional alternative translation initiation sites then it would be expected that these sites contain features that favour translation initiation, such as stem-loop structures. Therefore, the CPR5 mRNA sequence was further computationally analysed. Figure 1 shows the

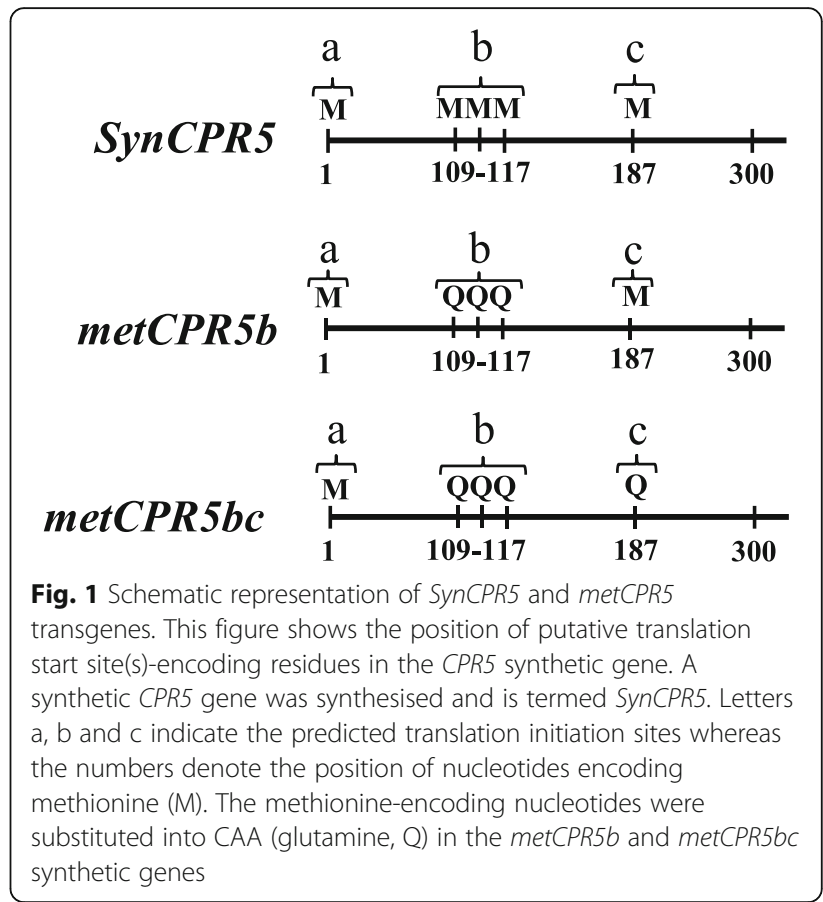

CPR5 mRNA sequence and the positions of the in-frame start codons. A closer view of the second putative site (site b) shows that it consists of three in-tandem start codons, 109 nt downstream of the first start codon. The third site occurs $187 \mathrm{nt}$ downstream of the first site. Furthermore, the CPR5 coding sequence is predicted to contain four stem-loop structures in the first 190 nucleotides (Figure S1). Of these stem-loops, the first is present between the first and second putative start site. This stem-loop appears to be strong as $50 \%$ of the associated nuceleotides involved in the putative stem formation are guanine and cytosine. Notably, the second putative start site is predicted to be part of a stem-loop. Each of the putative translation start sites is followed by a stem-loop structure (Figure S1). Additionally, putative favourable residues for translation initiation such as adenine or guanine are also present at the expected positions $(-3,+3$ and +4$)$ of each putative initiation site (Figure S1). These results are consitent with the model that the CPR5 transcript contains multiple translation initiation sites.

\section{Construction of a synthetic CPR5 gene with mutated start codons}

Complementation of $\operatorname{cpr} 5$ mutants with the wild type CPR5 gene rescues the compromised phenotypes associated with the mutation [18]. Therefore, we aimed to test the role of the putative alternative start sites in CPR5 function by complementing cpr5-2 plants with synthetic CPR5 constructs carrying mutations in the putative sites. In order to distinguish the complementing transgenic 
transcripts from cpr5-2 transcripts, a number of nucleotide changes were introduced in the synthetic CPR5 coding sequence by codon optimization. The codon optimized synthetic version of the CPR5 gene was termed SynCPR5 (Figs. 1 and S2) and constructs in which the putative start sites were mutated individually as well as collectively were called metCPR5 $b$ and metCPR5bc. The metCPR $5 b$ RNA translates into a CPR 5 protein with the three methionines of the second putative start site converted into glutamine residues. Glutamine was chosen for substitution since it resembles methionine in size and is presumed to have limited impact on protein tertiary structure [19]. The metCPR5bc RNA has the third putative start site mutated in addition to the second (Fig. 1). Thus, these synthetic constructs will allow the separate detection of endogenous cpr5-2 mRNA from the mRNA derived from the synthetic constructs in cpr5-2 lines complemented with the synthetic CPR5 genes.

CPR5 native promoter is able to drive SynCPR5 expression A number of studies have used the Cauliflower Mosaic Virus $35 S$ promoter to drive expression of the CPR5 gene $[13,20,21]$. Here, we expressed SynCPR5 from the native CPR5 promoter to mimic expression of the wild type CPR5 gene. Actual levels of SynCPR5 transcripts in transgenic lines were, however, expected to be lower than in the wild type, wild-type-like or higher than in the wild-type due to position effects $[22,23]$. A $1.5-\mathrm{kb}$ upstream fragment of the CPR5 gene (AT5G64930) was fused with the SynCPR5 coding sequence, and the whole cassette was transferred into cpr5-2 plants. Following successful SynCPR5 transformation, SynCPR5 expression of three independent homozygous complemented lines was compared with CPR5 expression in the wild type Col-0 and mutant cpr5-2 lines. Relative transcript abundance of SynCPR5, CPR5 and cpr5-2 genes was quantified using SynCPR5, CPR5 and cpr5-2 gene-specific primer sets (Figure S2 and Table S1), as described in Methods. As shown in Fig. 2, the relative SynCPR5 transcript abundance varied between the independent SynCPR5 lines. SynCPR5L1 showed wild type-like expression, whereas SynCPR5L2 and SynCPR5L3 displayed SynCPR5 transcript abundance higher than the wild type. To summarise, these results establish that the $1.5-\mathrm{kb}$ native CPR5 promoter was able to drive $S y n C P R 5$ expression in transgenic Arabidopsis plants.

\section{Transgenic metCPR5 lines exhibit bigger leaves than SynCPR5 and wild type lines}

Next, the metCPR5b and metCPR5bc constructs were transferred to cpr5-2 plants to determine the effect of mutated putative translation initiation sites on CPR5 activity. Three independent homozygous lines for each construct were selected for analysis and relative metCPR5 levels measured. As shown in Fig. 2, all metCPR5 $b$ lines displayed higher mRNA levels than wild type, while metCPR5bc lines showed wild type-like (Col-0) transcript abundances. During growth of the

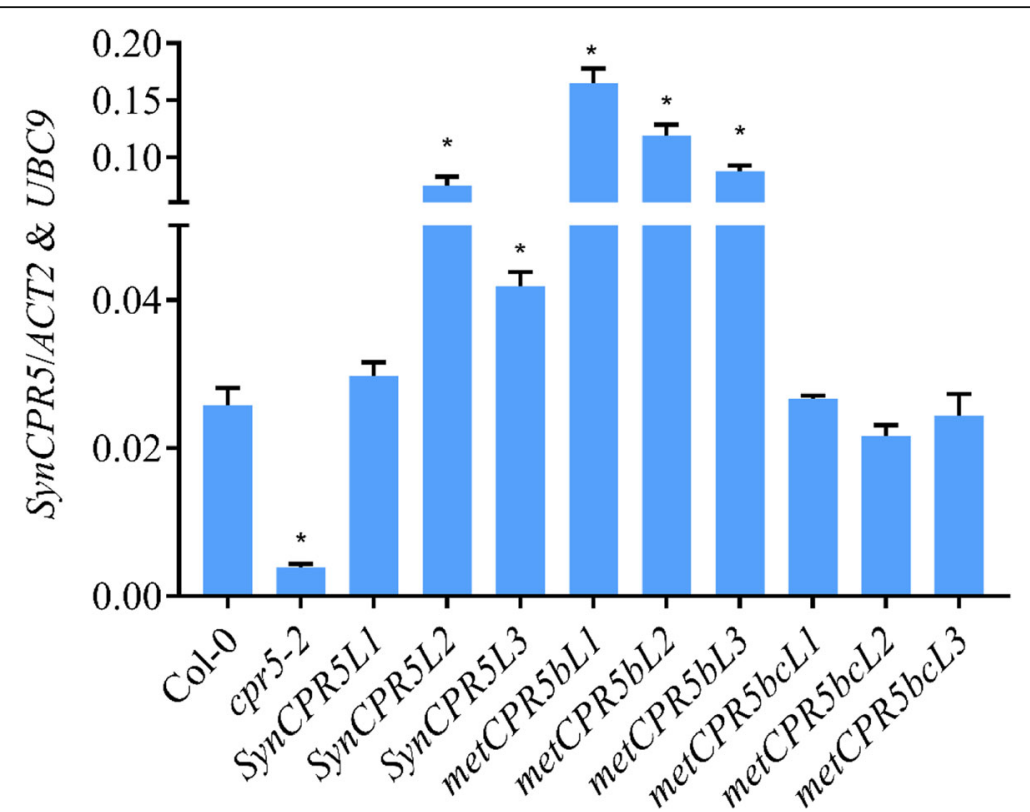

Fig. 2 Quantification of SynCPR5 transcripts in SynCPR5 and metCPR5 plants. Quantification of transcript abundance in SynCPR5, Col-0 and CPr5-2 plants using SynCPR5, CPR5 and cpr5-2 specific sets of primers. Three biological and three technical replicates were included, and values were normalized to the average expression values of ACT2 and UBC9 housekeeping genes. Error bars represent the standard error, and asterisks indicate significant difference from Col-0 at $p<0.05$ (Student's $t$-test) 
different transgenic and control wild-type and cpr5-2 plant lines grown under same conditions, we noted that the leaves of metCPR5 plants appeared to be bigger than those of the wild type (Fig. 3). This prompted us to quantify leaf sizes of the different plant lines. Plants were grown for 31 days at standard growth conditions and the leaf sizes of the third to fifth leaf pairs were measured as described in Methods. As shown in Figs. 3 and 4, the leaf areas of the SynCPR5 lines were indifferent from those of the wild type. In contrast, the leaf areas of the third (fifth and sixth rosette leaves), fourth (seventh and eighth rosette leaves) and fifth (ninth and tenth rosette leaves) leaf pairs of metCPR5 $b$ plants were significantly larger than those of wild-type and SynCPR5 lines (Fig. 4). Furthermore, the leaf areas of the fifth leaf pair of metCPR $5 b c$ plants were larger than those of wild type and SynCPR5 plants. In addition to leaf area, we determined the sizes of epidermal pavement cells. The cells of metCPR $5 b c$ plants were significantly bigger than those of the wild type $(p<0.05$; Student's $t$-test) (Figure S3).
Thus, these results indicate that the second putative alternative translation start site restricts leaf size.

\section{metCPR5b and metCPR5bc leaves have higher ploidy levels than wild type}

Leaf size is positively correlated with ploidy levels [24]. In $A$. thaliana, leaves generally undergo three to four rounds of endoreduplication and consequently contain nuclear populations of DNA content up to 16 or $32 \mathrm{C}$ [24]. We hypothesised that metCPR5 lines, having bigger leaves, have higher ploidy levels than wild type. To test this hypothesis, the third and the fourth leaves from 24day-old (24 DAS) metCPR5, SynCPR5 and wild type plants were harvested and nuclei were extracted for ploidy analysis (Fig. 5). Flow cytometry of the isolated nuclei showed that two of the SynCPR5 lines had ploidy levels indifferent from the wild type, while one had higher $2 \mathrm{C}$ and $4 \mathrm{C}$ levels, but lower $8 \mathrm{C}$ levels. Most metCPR5 lines had similar $2 \mathrm{C}$, but higher $4 \mathrm{C}$ levels than the wild type. Interestingly, all metCPR5 lines displayed

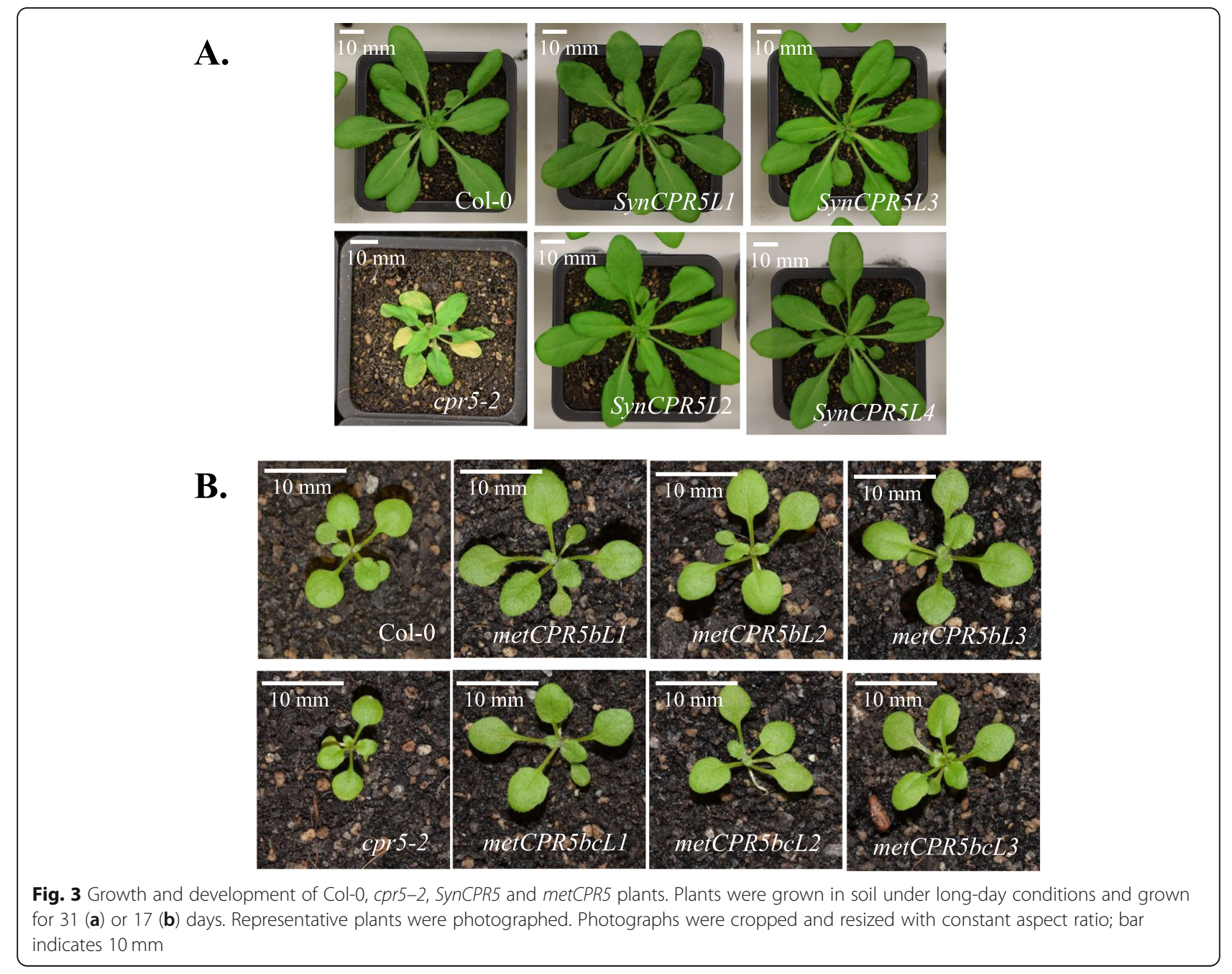




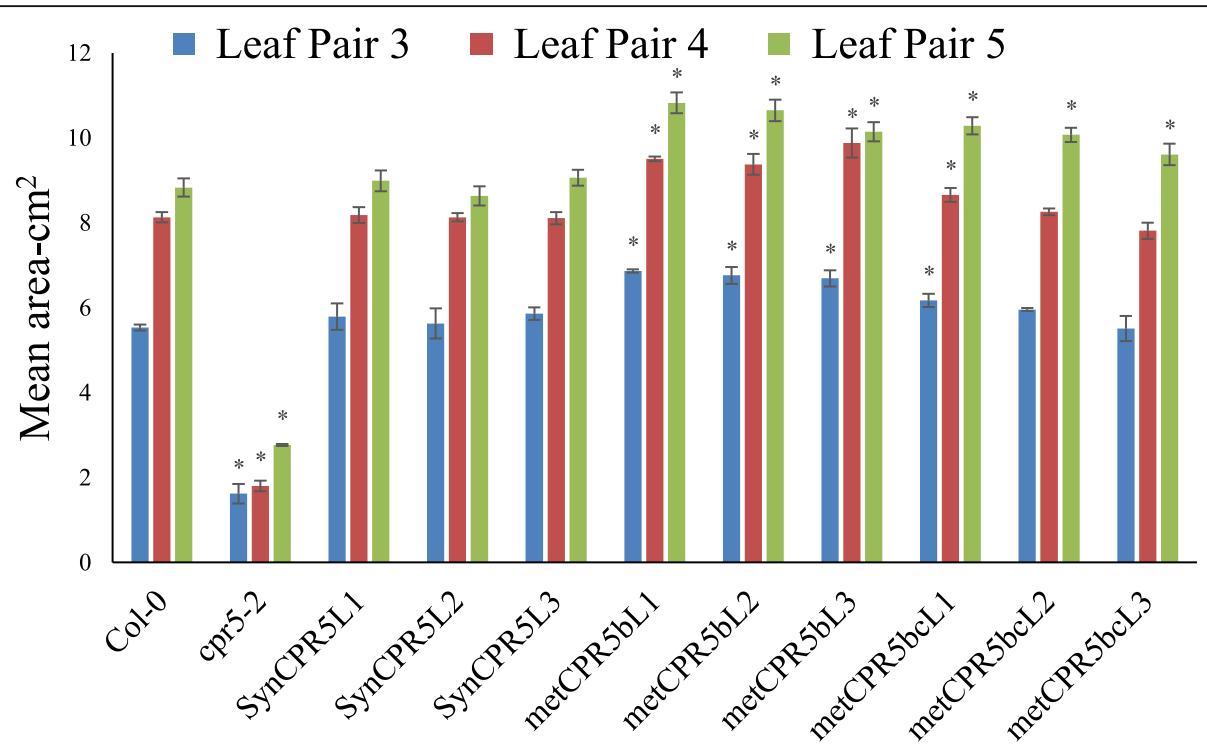

Fig. 4 Mean leaf area of rosette leaf pairs. Mean leaf areas of third, fourth and fifth rosette leaf pairs at 31 DAS. The leaves were removed from five different plants of the same line and leaf area was measured as described in Methods. Error bars represent the standard error, and asterisks indicate significant difference from Col-0 at $p<0.05$ (Student's $t$-test)

lower $8 \mathrm{C}$ levels and in addition, a $16 \mathrm{C}$ population was found (Fig. 5), while 16C nuclei were not detected in the other lines. These results support the hypothesis that the enlarged leaves are caused by increased ploidy levels in metCPR5 lines.

\section{metCPR5 plants conferred enhanced susceptibility to $P$.} syringae

Since cpr5-2 mutants display reduced leaf size combined with enhanced resistance to infection from Pseudomonas syringae pv. tomato DC3000 (Pst DC3000), we hypothesised that the larger leaves of metCPR5 plants may coincide with increased susceptibility to Pst DC3000. Thus, metCPR5 plants were grown in neutral days (12:12 light: dark) conditions. Leaves from 4- to 5-week-old plants were infiltrated with Pst DC3000, and susceptibility was scored by measuring the number of colony forming units (cfu) as described (52). As shown in Fig. 6, the $m e t C P R 5 b$ and metCPR $5 b c$ lines displayed significantly

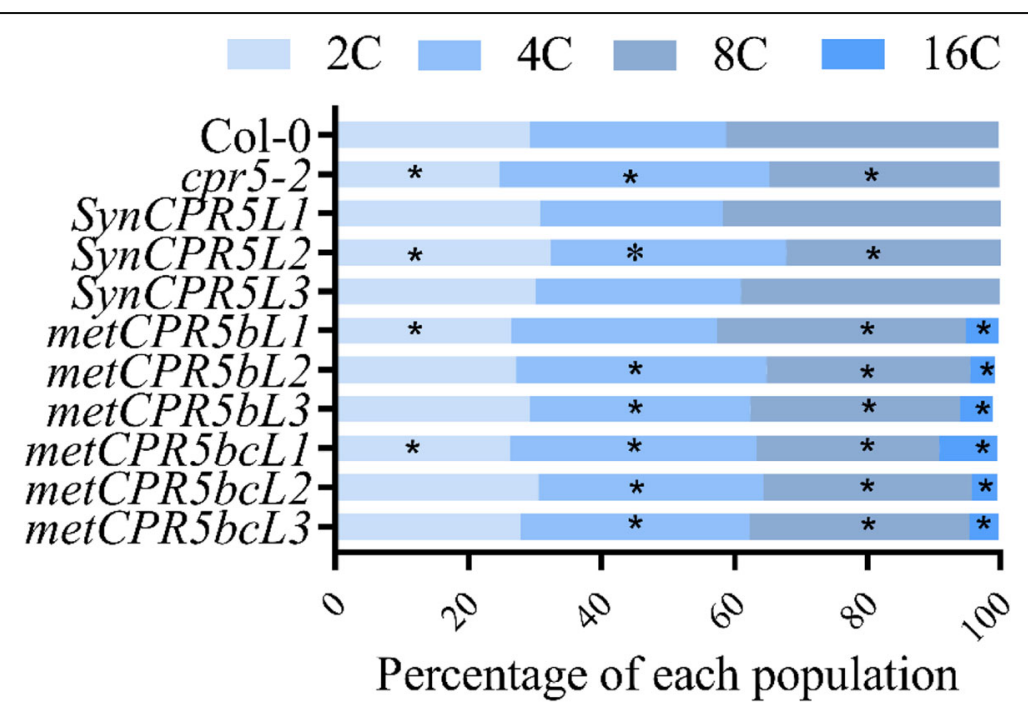

Fig. 5 Ploidy levels of nuclei isolated from leaf tissue. Figure shows percentages of nuclei populations extracted from leaf cells of Col-0, cpr5-2, SynCPR5 and metCPR5 plants. The nuclei were extracted from leaf cells of 24-old-plants and analysed by flow cytometry as described in Methods. Percentages of every population from each sample were calculated and average (percentage) values are shown in the graph. Each value represents the mean of three values from leaves of three different plants of the same line. Asterisks indicate significant difference from Col-0 at $p<0.05$ (Student's t-test) 
higher susceptibility to Pst DC3000 compared to the wild type. In contrast, SynCPR5 lines displayed susceptibility to Pst DC3000 in a wild type manner except SynCPR5L2, which displayed higher susceptibility than the wild type. In conclusion, metCPR5 plants, having larger leaves than wild type, show reduced resistance to Pst DC3000 infection.

\section{metCPR5 lines have variable $P R 1$ levels}

Expression of SA-mediated disease resistance marker gene PATHOGENESIS RELATED GENE1 (PR1) is enhanced in $c p r 5-2$ mutants and correlates with resistance [9]. Therefore, it was expected that metCPR5 lines have reduced PR1 levels. The transcript abundance of PR1 was quantified from uninfected wild type, cpr5-2, SynCPR5 and metCPR5 lines using real-time qRT-PCR. As shown in Fig. 7, cpr5-2 plants showed greatly increased PR1 levels. However, most metCPR5 lines displayed $P R 1$ transcript levels similar to those of the wild type. The exceptions were metCPR5bL3 and metCPR $5 b c L 1$ lines, which had lower and higher PR1 levels, respectively. Remarkably, all $\operatorname{Syn} C P R 5$ lines exhibited increased PR1 levels compared to wild type (Fig. 7).

\section{Discussion}

\section{Role of CPR5 in growth and resistance regulation}

Plants balance their resource allocation between resistance and growth to limit negative impacts on plant growth [26, 27]. To further minimise cost, plants have developed distinct, highly orchestrated defense systems for different types of biotic and abiotic stresses [29-30]. For example, salicylic acid (SA)-mediated resistance is activated in response to biotrophic pathogens, whereas JA/ET-mediated resistance is induced upon necrotrophic pathogen invasion [31]. The constitutive activation of defense systems results in slow or reduced growth and development $[28,29,32]$. The phenotype of the cpr5 mutant, i.e. compromised growth combined with increased resistance against the pathogens $P$. syringae and Hyaloperonospora parasitica [9] is consistent with this notion and suggests that resistance in cpr5 is gained at the cost of growth. This is also in agreement with the finding that a large number of genes involved in auxin and gibberellin (GA) biosynthesis and signalling are repressed in $\operatorname{cpr} 5$ [17]. Perhaps the simplest explanation of the phenotype is that CPR5 functions as a suppressor of resistance. A number of observations suggest that this is the case: the upregulation of Pathogenesis-Related genes (PR1 and PR5) and Plant Defensin 1.2 (PDF1.2), the formation of HR-like lesions and the higher SA levels in cpr5 plants $[8,9,11,14]$. Furthermore, the deregulation of cellular redox balance in cpr5 plants has been proposed to be involved in the induction of lesions and early leaf senescence [33], suggesting that CPR5 may supress resistance by regulating ROS levels [16]. Similarly, the hypersensitive response of $c p r 5$ plants to the hormones SA, JA, ABA and ET [13, 20] indicates that CPR5 may regulate SA- and JA/ET-mediated resistance by suppressing the magnitude of these hormone responses. CPR5 has also been shown to suppress a set

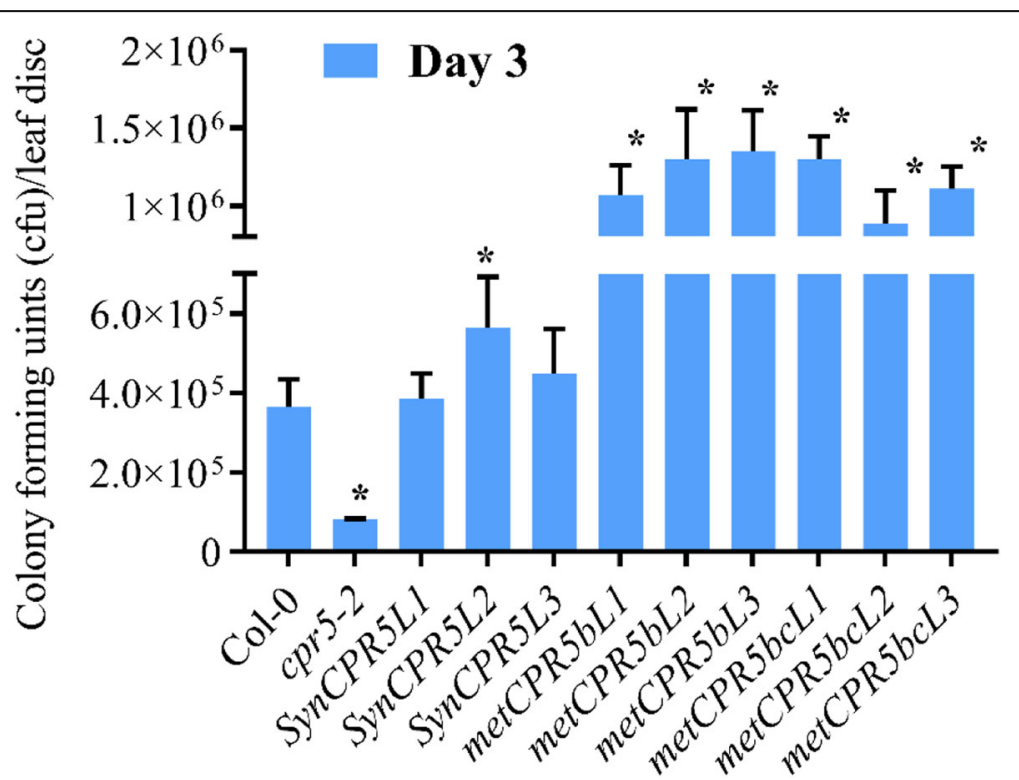

Fig. 6 Pst DC3000 infection growth assay. Three leaf discs were cut from randomly selected leaves of three plants that were inoculated with Pst DC3000 and bacteria were extracted as described in [25]. Pst DC3000 bacteria were extracted from leaf discs (1 $\left.\mathrm{cm}^{2}\right)$ and plated onto agar plates. Colony forming units (cfu) were measured after 3 days of growth. The error bars represent the standard error of the mean and asterisks indicate significant difference from Col-0 at $p<0.05$ (Student's $t$-test) 


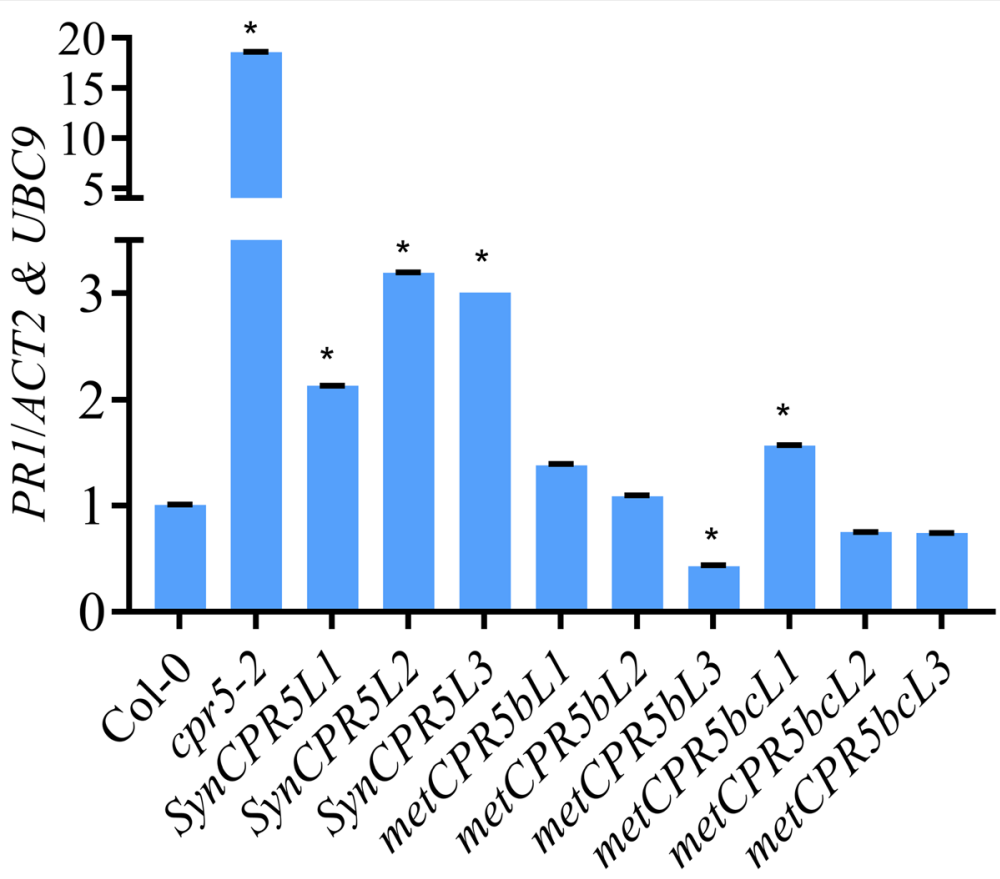

Fig. 7 Relative expression levels of PR1 in SynCPR5 plants. Total RNA was extracted from whole rosettes of 31-day-old plants, and relative levels of PR1 mRNA were measured. Three biological and three technical replicates were included, and values were normalized to the average expression values of $A C T 2$ and UBC9 housekeeping genes. Error bars represent the standard error of the mean and asterisks indicate significant difference from Col-0 at $p<0.05$ (Student's t-test)

of genes involved in the biosynthesis of the cell wall [21], which functions as a first barrier against pathogen infection [34]. Nevertheless, aberrant trichomes, smaller epidermal cells and reduced ploidy levels clearly show that CPR5 is involved in growth regulation as well and it has been suggested that activation of resistance in cpr5 could be the consequence of growth defects [35], including abnormal cell wall synthesis [36].

We show here that nucleotide changes in metCPR5 result in plants that grow larger leaves than the wild type, at the expense of resistance to Pst DC3000. The mutated base pairs, rather than mRNA abundance, appear to be critical because the phenotypes correlated with the type of mutations and not the mRNA abundance. This is also consistent with previous results where overexpression of the wild type CPR5 gene using the $35 \mathrm{~S}$ promoter resulted in 1000-fold increase in gene expression, without similar larger leaves and decreased resistance being reported [13]. This suggests that the observed phenotype is a result of structural properties of the metCPR 5 protein. However, we cannot exclude the possibility that the methionine-encoding nucleotides somehow limit protein translation which is overcome by the metCPR5 mutations, leading to higher protein levels. The results are furthermore consistent with the model that CPR5 does not simply function as a repressor of disease resistance, but it regulates the balance between growth and resistance, whereby structural properties - present at the $\mathrm{N}$ - terminus - limit the extent by which CPR5 can favour growth over resistance.

How does CPR5 control the growth-resistance balance?

The mechanism of how plants regulate the balance between growth and resistance is poorly understood due to its complex nature and involvement of a large number of growth- and resistance-related genes or hormones. For example, auxins, brassinosteroids, cytokinins, gibberellins, abscisic acid, ethylene, and jasmonates, have all been shown to be involved in the regulation of plant growth and immunity [26, 37-43]. Notably, the levels or signalling pathways of many of these hormones are affected in cpr5 mutants as well: the levels of salicylic acid (SA) and jasmonic acid (JA) are higher in cpr5 plants, cpr5 plants have perturbations in SA, JA, ABA and ET signalling pathways [13], and cpr5 plants are hypersensitive to exogenous SA, ABA, JA and ET [13, 15]. Recently, Meng et al. (2017) showed that CPR5 regulates growth and stress response-tradeoff through the modulation of SA and the unfolded protein response. In addition, a large number of growth promoting genes such as auxin and GA response genes are suppressed in cpr5 mutants [17]. Nuclear accumulation of DELLA proteins leads to the repression on GA signalling, whereas their degradation release repression on GA biosynthesis signalling [44]. The misregulation of DELLA target genes in cpr5 plants indicates that GA levels could be affected 
in cpr5 plants as well [17]. Taken together, these results indicate that altered levels or signalling of the abovementioned hormones may affect growth and resistance in $\operatorname{cpr} 5$ mutant plants. This is in line with our hypothesis that CPR5 plays a central role in regulating the balance between growth and resistance by mediating growth and resistance hormones. Recent discoveries of how CPR5 functions provides a means to propose how CPR5 controls growth and resistance: CPR5 is one of the nucleoporins which function as part of the Nuclear Pore Complex (NPC) and inhibit the activities of NPC and the cyclin-dependent kinases (CKIs) under stress-free conditions $[14,15,17]$. As a consequence of conformational changes in cpr 5 mutants or as a result of stress, CPR5-mediated gating is altered, allowing a number of molecules to enter the nucleus, leading to the activation of resistance genes [17]. Furthermore, CPR5 participates in NPC gating and acts as a selective barrier through the formation of CPR5-CPR5 homodimers [17]. The Nterminal region of CPR5 is crucial for CPR5-CPR5 homomeric interaction within the NPC and when this interaction was lost in the old1-l3 allele of CPR5 (G120D), proteins such as NPR1, JAZ1, and ABI5 were found to be aberrantly not retained in the cytoplasm [17]. These results are in general agreement with ours, which show that mutations that cause changes in the Nterminal part of the CPR5 protein can improve plant growth at the expense of disease resistance, possibly through altered CPR5-CPR5 homodimerization and CPR5-mediated NPC gating. Nevertheless, in addition to possible structural changes of metCPR 5 proteins, the nucleotide changes in metCPR5 transcripts may affect CPR5 function in other ways: the mutations remove possible alternative translation initiation sites and different alternatively translated CPR5 proteins may affect NPC gating. Indeed, in silico analysis of the CPR5 transcript identified potential RNA secondary structures nearby putative alternative translation start sites and similar secondary structures have been shown to be important for the activity of alternative translation start sites [45-49]. Alternatively, or in addition, the induced mutations may have affected the secondary structures themselves and as such may have modified translation.

\section{Conclusion}

Taken altogether, these results led us to propose that the changed nucleotides in metCPR5 could have altered CPR5-CPR5 homodimerization and consequently CPR5mediated NPC gating. Any structural difference(s) in the NPC complex could then affect the balance between growth and resistance by a changed gating of growth and/or resistance regulators.

\section{Methods}

\section{Plant materials and growth conditions}

Arabidopsis thaliana Columbia (CPR5 and cpr5-2) seeds were the same as used in [50] studies. Seeds were washed and disinfected according to the method described by [51] and imbibed in the dark for 3-4 days at $4{ }^{\circ} \mathrm{C}$. Imbibed seeds were grown on autoclaved Daltons Premium Seed Raising Mix ${ }^{\circ}$, New Zealand. Unless mentioned, the plants were grown at $22^{\circ} \mathrm{C}$ and $65 \%$ Relative Humidity $(\mathrm{RH})$ under long-day conditions (16 h light, 8 $\mathrm{h}$ dark). For Pseudomonas syringae bacterial infiltrations, plants were grown under short-day conditions $(10 \mathrm{~h}$ light, $14 \mathrm{~h}$ dark) at $21^{\circ} \mathrm{C}$ and $65 \% \mathrm{RH}$.

\section{Trichome counting, leaf and pavement cell area measurements}

Leaves were removed from plants grown and mounted on a glass slide. Number of appendages on each trichome was counted under a dissecting microscope. At 31 DAS (days after sowing), leaf pairs were removed from plants and fed onto a leaf area machine (LI-3050A/4, LICOR, USA) for the combined measurement of both leaves. Leaves were harvested in pairs: leaf 1 and 2 were considered as leaf pair 1 , leaf 3 and 4 as leaf pair 2, and so on. Leaf 3 from three different plants of the same transgenic line was imaged using a scanning electron microscope (FEI Quanta 200 Environmental Scanning Electron Microscope (SEM) with EDAX module) following manufacturer's instructions at Manawatu Microscopy Imaging Centre (MMIC), Massey University, Palmerston North. The images were processed and cell areas were measured individually by ImageJ (https://imagej.nih.gov/ij/).

\section{CPR5 gene synthesis and transformations}

The modified CPR5 gene variants, SynCPR5 (Figure S1) and metCPR5, were synthesized by GenScript (GenScript $^{\circ}$, USA). The CPR5 gene variants, along with a 1.5 $\mathrm{kb}$ native CPR5 promoter fragment, were cloned into pGreen0229 by GenScript. Sequence fidelity of cloned SynCPR5 and metCPR5 constructs was confirmed by Sanger sequencing and restriction digestion analyses. Plasmids were transformed into Agrobacterium tumefaciens GV3101, which was then used to transform cpr5-2 plants using floral dipping as described [52]. Seeds harvested from the first generation of the transformed plants were grown on soil beds for screening of positive transformants using BASTA $(125 \mathrm{mg} / \mathrm{L})$. Homozygous transformed lines were selected in subsequent generations. The presence of the transgene in transformed lines was confirmed by DNA sequencing, restriction digestion analyses and PCR using CPR5, SynCPR5 and metCPR5 transgene-specific sets of primers (Figure S1). 


\section{Ploidy level measurements, qRT-PCR data analyses and pathogenicity assays}

For flow cytometry, nuclei were isolated, stained and analysed as described [21]. Kinematic analyses (DNA content measurements) of the CPR5 transgenic lines, including Col-0 (CPR5) and cpr5-2, were carried out using a Sysmec Cyflow cytometer (Partec, CyFlow, USA) as per manufacturer's instructions. Total RNA was isolated from plants using Quick-RNA Miniprep kit (Zymo Research) and cDNA was prepared using Transcriptor First Strand cDNA Synthesis Kit (Roche). Transcript abundance was quantified using the LightCycler 480 RealTime PCR (Roche) system [25]. Transcript abundance and statistical analyses were performed using Microsoft Office Excel 2010 as described [53]. Pseudomonas syringae pv DC3000 pathogenicity assays were carried out as described [25].

\section{Supplementary information}

Supplementary information accompanies this paper at https://doi.org/10. 1186/s12870-020-02485-2.

Additional file 1: Figure S1: Positions of putative translation start sites and RNA stem-loop structures. Figure S2: Sequence comparison and positions of primers used for real-time qRT-PCR quantifications. Figure S3: Area of abaxial epidermal pavement cells. Table S1: List of primers.

\section{Abbreviations}

ABA: Abscisic acid; ABI5: ABA Insensitive 5; acd6: Accelerated cell death 6; AT3G18780: ACTIN 2; AT4G27960: Ubiquitin Conjugating Enzyme 9 (UBC9); AT5G64930: CPR5; CDNA: Complementary DNA; CK: Casein kinase; CKIs: Cyclindependent kinases; cpr1: Constitutive expresser of pathogenesis-related genes 1: cpr2 and cpr6: constitutive expresser of pathogenesis-related genes 1 and 6; CPR5: CONSTITUTIVE EXPRESSER of PATHOGENESIS-RELATED GENES 5; DAS: Days after sowing; eds5: Enhanced disease susceptibility 5; ET: Ethylene; hys 1: Hypersenescence 1; JA: Jasmonic acid; JAZ1: Jasmonate ZIM-domain 1; NLS: Nuclear localisation signal; NPC: Nuclear pore complex; npr1: Nonexpresser of pathogenesis-related genes 1; NPR1: NON-EXPRESSER OF PATHOGENESIS-RELATED GENES 1; old1: Onset of leaf death 1; PDF1.2: PLANT DEFENSIN 1.2; PR1: PATHOGENESIS RELATED GENE 1; RNA/DNA: Ribonucleic acid / deoxyribonucleic acid; SA: Salicylic acid; SynCPR5: Synthetic version of CPR5; TM domains: Transmembrane domains

\section{Acknowledgements}

We would like to thank Dr. Gill Norris, SFS, Massey University, Palmerston North for her help in selecting amino acids for metCPR5 mutants, Dr. Tina Sehrish, SFS, Massey University, Palmerston North, for technical support and helpful discussions and Niki Minards, Manawatu Microscopy Imaging Centre (MMIC), Massey University, for the scanning electron microscopy work.

\section{Authors' contributions}

MBF and PPD designed the project. MBF conducted all the experimental work. MBF, PPD, TSG and BMR analysed the data and wrote the manuscript. All authors read and approved the manuscript.

\section{Funding}

MBF is a recipient of a Higher Education Commission (HEC), Pakistan, scholarship. EU funding of FP7-IRSES/ RSNZ grant \# 612630 to PPD. EU Horizon 2020 research and innovation programme, project PlantaSYST to TSG and BMR (SGA-CSA No. 739582 under FPA No. 664620). TSG acknowledges the BG05M2OP001-1.003-001-C01 project, financed by the European Regional Development Fund through the Bulgarian "Science and Education for Smart Growth" Operational Programme. The funding agencies were not involved in the experimental design, data collection and analysis or preparation of the manuscript.

\section{Availability of data and materials}

All data generated and/or analysed during this study are included in this published article and its supplementary information files. The datasets used and/or analysed during the current study are available from the corresponding author on reasonable request.

Ethics approval and consent to participate

Not applicable.

Consent for publication

Not applicable.

\section{Competing interests}

The authors declare that they have no competing interests.

\section{Author details}

${ }^{1}$ School of Fundamental Sciences, Massey University, Palmerston North, New Zealand. ${ }^{2}$ Center of Plant Systems Biology and Biotechnology, 4000 Plovdiv, Bulgaria. ${ }^{3}$ Department of Molecular Biology, Institute of Biochemistry and Biology, University of Potsdam, 14476 Potsdam, Germany.

Received: 7 January 2020 Accepted: 9 June 2020

Published online: 29 June 2020

\section{References}

1. Dangl JL, Dietrich RA, Richberg MH. Death Don't have no mercy: cell death programs in plant-microbe interactions. Plant Cell. 1996;8(10):1793-807.

2. Cao H, Bowling SA, Gordon AS, Dong X. Characterization of an Arabidopsis mutant that is nonresponsive to inducers of systemic acquired resistance. Plant Cell. 1994;6(11):1583.

3. Delaney TP, Friedrich L, Ryals JA. Arabidopsis signal transduction mutant defective in chemically and biologically induced disease resistance. Proc Natl Acad Sci U S A. 1995:92(14):6602-6.

4. Shah J, Kachroo P, Klessig DF. The Arabidopsis ssil mutation restores pathogenesis-related gene expression in npr 1 plants and renders defensin gene expression salicylic acid dependent. Plant Cell. 1999;11(2):191-206.

5. Rogers EE, Ausubel FM. Arabidopsis enhanced disease susceptibility mutants exhibit enhanced susceptibility to several bacterial pathogens and alterations in PR-1 gene expression. Plant Cell. 1997:9(3):305-16.

6. Nawrath C, Métraux JP. Salicylic acid induction-deficient mutants of Arabidopsis express PR-2 and PR-5 and accumulate high levels of camalexin after pathogen inoculation. Plant Cell. 1999;11(8):1393-404.

7. Bowling SA, Guo A, Cao H, Gordon AS, Klessig DF, Dong X. A mutation in Arabidopsis that leads to constitutive expression of systemic acquired resistance. Plant Cell. 1994;6(12):1845-57.

8. Clarke JD, Volko SM, Ledford H, Ausubel FM, Dong X. Roles of salicylic acid, jasmonic acid, and ethylene in cpr-induced resistance in Arabidopsis. Plant Cell. 2000:12(11):2175-90.

9. Bowling SA, Clarke JD, Liu Y, Klessig DF, Dong X. The cpr5 mutant of Arabidopsis expresses both NPR1-dependent and NPR1-independent resistance. Plant Cell. 1997:9(9):1573-84.

10. Jing HC, Sturre MJ, Hille J, Dijkwel PP. Arabidopsis onset of leaf death mutants identify a regulatory pathway controlling leaf senescence. Plant J. 2002;32(1):51-63.

11. Yoshida S, Ito M, Nishida I, Watanabe A. Identification of a novel gene HYS1/ CPR5 that has a repressive role in the induction of leaf senescence and pathogen-defence responses in Arabidopsis thaliana. Plant J. 2002;29(4):42737.

12. Borghi M, Rus A, Salt DE. Loss-of-function of Constitutive Expresser of Pathogenesis Related Genes 5 affects potassium homeostasis in Arabidopsis thaliana. PLoS One. 2011;6(10):e26360.

13. Jing HC, Anderson L, Sturre MJ, Hille J, Dijkwel PP. Arabidopsis CPR5 is a senescence-regulatory gene with pleiotropic functions as predicted by the evolutionary theory of senescence. J Exp Bot. 2007:58(14):3885-94.

14. Wang S, Gu Y, Zebell SG, Anderson LK, Wang W, Mohan R, et al. A noncanonical role for the CKI-RB-E2F cell-cycle signaling pathway in plant effector-triggered immunity. Cell Host Microbe. 2014;16(6):787-94. 
15. Wang $\mathrm{S}$. The CPR5-CKI signaling pathway plays a central role in integrating plant stress responses. Can J Plant Sci. 2017;97(5):767-70.

16. Jing HC, Dijkwel PP. CPR5: a Jack of all trades in plants. Plant Signal Behav. 2008;3(8):562-3.

17. Gu Y, Zebell Sophia G, Liang Z, Wang S, Kang B-H, Dong X. Nuclear pore permeabilization is a convergent signaling event in Effector-Triggered Immunity. Cell. 2016;166(6):1526-38 e11.

18. Liu YG, Shirano Y, Fukaki H, Yanai Y, Tasaka M, Tabata S, et al. Complementation of plant mutants with large genomic DNA fragments by a transformation-competent artificial chromosome vector accelerates positional cloning. Proc Natl Acad Sci U S A. 1999;96(11):6535-40.

19. Xiao Z, Liu X, Henis YI, Lodish HF. A distinct nuclear localization signal in the $\mathrm{N}$ terminus of Smad 3 determines its ligand-induced nuclear translocation. Proc Natl Acad Sci U S A. 2000;97(14):7853-8.

20. Gao G, Zhang S, Wang C, Yang X, Wang Y, Su X, et al. Arabidopsis CPR5 independently regulates seed germination and postgermination arrest of development through LOX pathway and ABA signaling. PLoS One. 2011; 6(4):e19406.

21. Perazza D, Laporte F, Balague C, Chevalier F, Remo S, Bourge M, et al. GeBP/ GPL transcription factors regulate a subset of CPR5-dependent processes. Plant Physiol. 2011;157(3):1232-42.

22. Peach $C$, Velten J. Transgene expression variability (position effect) of CAT and GUS reporter genes driven by linked divergent T-DNA promoters. Plant Mol Biol. 1991;17(1):49-60.

23. Gelvin SB. Agrobacterium-mediated plant transformation: the biology behind the "gene-jockeying" tool. Microbiol Mol Biol Rev. 2003;67(1):16-37.

24. Sugimoto-Shirasu K, Roberts K. "big it up": endoreduplication and cell-size control in plants. Curr Opin Plant Biol. 2003;6(6):544-53.

25. Tahir J, Watanabe M, Jing H-C, Hunter DA, Tohge T, Nunes-Nesi A, et al. Activation of R-mediated innate immunity and disease susceptibility is affected by mutations in a cytosolic O-acetylserine (thiol) lyase in Arabidopsis. Plant J. 2013;73(1):118-30.

26. Lozano-Durán R, Zipfel C. Trade-off between growth and immunity: role of brassinosteroids. Trends Plant Sci. 2015;20(1):12-9.

27. Meng Z, Ruberti C, Gong Z, Brandizzi F. CPR5 modulates salicylic acid and the unfolded protein response to manage tradeoffs between plant growth and stress responses. Plant J. 2017:89(3):486-501.

28. Walters $\mathrm{D}$, Heil M. Costs and trade-offs associated with induced resistance. Physiol Mol Plant Pathol. 2007;71(1):3-17.

29. Huot B, Yao J, Montgomery BL, He SY. Growth-Defense Tradeoffs in Plants: A Balancing Act to Optimize Fitness. Mol Plant (Oxford University Press / USA). 2014;7(8):1.

30. Smakowska E, Skibior-Blaszczyk R, Czarna M, Kolodziejczak M, KwasniakOwczarek M, Parys K, et al. Lack of FTSH4 protease affects protein carbonylation, mitochondrial morphology, and phospholipid content in mitochondria of Arabidopsis: new insights into a complex interplay. Plant Physiol. 2016;171(4):2516.

31. Caarls L, Pieterse CMJ, Van Wees SCM. How salicylic acid takes transcriptional control over jasmonic acid signaling. Front Plant Sci. 2015;6:170.

32. Kempel A, Schädler M, Chrobock T, Fischer M, van Kleunen M. Tradeoffs associated with constitutive and induced plant resistance against herbivory. Proc Natl Acad Sci. 2011;108(14):5685.

33. Jing HC, Hebeler R, Oeljeklaus S, Sitek B, Stühler K, Meyer HE, et al. Early leaf senescence is associated with an altered cellular redox balance in Arabidopsis cpr5/old1 mutants. Plant Biol. 2008;10(s1):85-98.

34. Engelsdorf T, Gigli-Bisceglia N, Veerabagu M, McKenna JF, Augstein F, van der Does D, et al. Pattern-Triggered Immunity and cell wall integrity maintenance jointly modulate plant stress responses. bioRxiv. 2017:130013.

35. Kirik V, Bouyer D, Schobinger U, Bechtold N, Herzog M, Bonneville JM, et al. CPR5 is involved in cell proliferation and cell death control and encodes a novel transmembrane protein. Curr Biol. 2001;11(23):1891-5.

36. Brininstool G, Kasili R, Simmons LA, Kirik V, Hulskamp M, Larkin JC. Constitutive Expressor of Pathogenesis-Related Genes 5 affects cell wall biogenesis and trichome development. BMC Plant Biol. 2008;8:58.

37. Llorente F, Muskett P, Sanchez-Vallet A, Lopez G, Ramos B, Sanchez-Rodriguez C, et al. Repression of the auxin response pathway increases Arabidopsis susceptibility to necrotrophic fungi. Mol Plant. 2008;1(3):496-509.

38. Kazan K, Manners JM. Linking development to defense: auxin in plantpathogen interactions. Trends Plant Sci. 2009;14(7):373-82.
39. Choi J, Huh SU, Kojima M, Sakakibara H, Paek K-H, Hwang I. The cytokininactivated transcription factor ARR2 promotes plant immunity via TGA3/NPR1pependent salicylic acid signaling in Arabidopsis. Dev Cell. 2010;19(2):284-95.

40. Choi J, Choi D, Lee S, Ryu C-M, Hwang I. Cytokinins and plant immunity: old foes or new friends? Trends Plant Sci. 2011;16(7):388-94.

41. Denancé N, Sánchez-Vallet A, Goffner D, Molina A. Disease resistance or growth: the role of plant hormones in balancing immune responses and fitness costs. Front Plant Sci. 2013;4:155.

42. Claeys H, De Bodt S, Inzé D. Gibberellins and DELLAs: central nodes in growth regulatory networks. Trends Plant Sci. 2014;19(4):231-9.

43. Song S, Huang H, Gao H, Wang J, Wu D, Liu X, et al. Interaction between MYC2 and ETHYLENE INSENSITVEE 3 modulates antagonism between jasmonate and ethylene signaling in Arabidopsis. Plant Cell. 2014;26(1):263-79.

44. Wang F, Zhu D, Huang X, Li S, Gong Y, Yao Q, et al. Biochemical insights on degradation of Arabidopsis DELLA proteins gained from a cell-free assay system. Plant Cell. 2009;21 (8):2378-90.

45. Chabregas SM, Luche DD, Van Sluys MA, Menck CF, Silva-Filho MC. Differential usage of two in-frame translational start codons regulates subcellular localization of Arabidopsis thaliana TH11. J Cell Sci. 2003;116(Pt 2):285-91.

46. Sunderland PA, West CE, Waterworth WM, Bray CM. Choice of a start codon in a single transcript determines DNA ligase 1 isoform production and intracellular targeting in Arabidopsis thaliana. Biochem Soc Trans. 2004;32(Pt 4):614-6.

47. Christensen AC, Lyznik A, Mohammed S, Elowsky CG, Elo A, Yule R, et al. Dual-domain, dual-targeting organellar protein presequences in Arabidopsis can use non-AUG start codons. Plant Cell. 2005;17(10):2805-16.

48. Wamboldt Y, Mohammed S, Elowsky C, Wittgren C, de Paula WB, Mackenzie SA. Participation of leaky ribosome scanning in protein dual targeting by alternative translation initiation in higher plants. Plant Cell. 2009;21(1):157-67.

49. Asano K. Why is start codon selection so precise in eukaryotes? Translation. 2014;2(1):e28387.

50. Boch J, Verbsky ML, Robertson TL, Larkin JC, Kunkel BN. Analysis of resistance gene-mediated defense responses in Arabidopsis thaliana plants carnying a mutation in CPR5. Mol Plant-Microbe Interact. 1998;11(12):1196-206.

51. Weigel D, Glazebrook J. Arabidopsis: A Laboratory Manual, vol. 354. Cold Spring Harbor: Laboratory Press; 2002.

52. Zhang $X$, Henriques R, Lin SS, Niu QW, Chua NH. Agrobacterium-mediated transformation of Arabidopsis thaliana using the floral dip method. Nat Protoc. 2006;1 (2):641-6.

53. Pfaffl MW, Tichopad A, Prgomet C, Neuvians TP. Determination of stable housekeeping genes, differentially regulated target genes and sample integrity: BestKeeper - excel-based tool using pair-wise correlations. Biotechnol Lett. 2004;26(6):509-15.

\section{Publisher's Note}

Springer Nature remains neutral with regard to jurisdictional claims in published maps and institutional affiliations.

Ready to submit your research? Choose BMC and benefit from:

- fast, convenient online submission

- thorough peer review by experienced researchers in your field

- rapid publication on acceptance

- support for research data, including large and complex data types

- gold Open Access which fosters wider collaboration and increased citations

- maximum visibility for your research: over $100 \mathrm{M}$ website views per year

At BMC, research is always in progress.

Learn more biomedcentral.com/submissions 